\title{
An Improved Ant Colony Based Algorithm for Dynamic Routing and Wavelength Assignment Scheme in Optical Networks
}

\author{
Jianhua Shen \\ College of Communication and Information \\ Engineering \\ Nanjing University of Posts \&Telecommunications \\ Nanjing,China, 210003
}

\author{
Xi Cheng \\ College of Communication and Information \\ Engineering \\ Nanjing University of Posts \&Telecommunications \\ Nanjing,China, 210003
}

\begin{abstract}
Dynamic routing and wavelength assignment (RWA) is a key problem in intelligent optical networks. This paper presents an improved ant colony algorithm to solve the RWA problem which introduced link idle as constraint into the ant transition probability. At the same time, the random disturbance is added to prevent premature convergence into local optimal path. Simulation shows that the modified algorithm decreases the blocking probability and increases the resources utilization comparing with the traditional Dijkstra+FF algorithm, respectively.
\end{abstract}

Keywords-Intelligent Optical Network;Routing and Wavelength Assignment;Ant Colony Algorithm;Blocking Probability; Resource Utilization

\section{INTRODUCTION}

With the fast development of Internet and various IPbased services, traditional telecommunications network is undergoing tremendous changes. Many new interactive and real-time applications have been shown a great challenge for the traditional transport network. To meet these requirements, optical networks have achieved enormous progress especially the enhancement of automatic resource discovery and dynamic bandwidth allocation capabilities. Traditional optical networks depend on the network management system to configure network resources and service statically which can not satisfy the increasing new service demands. Thus, the new generation intelligent optical networks (ION) come into being which supporting many new characteristics such as high capacity, scalable, reconfigurable, survivability, etc. Compared with traditional optical networks, the main prominent merit of ION is the introduction of a distributed control plane by which some essential functions defined in ION can be achieved including automatic resource discovery, dynamic connection control and link management, etc[1]. When a client layer service arrives, it requets the ION to calculate route and assign a available wavelength which is called routing and wavelength assignment (RWA) problem in ION.

The essential issues of RWA problem is how to calculate and select an endto-end route (path), then assign available wavelength while the network topology and traffic load varying continuously. Considering the complexity of the optical network topology and differentation of traffic load, it is quite difficult to solve RWA problem especially when the available number of wavelengths on each link is limited. There are three major connection types in ION, i.e. Permanent Connection (PC), Soft Permanent Connection (SPC) and Switching Connecting (SC), and only the SC is a control plane based connection which belongs to dynamic RWA problem. Dynamic RWA needs to complete route calculating and wavelength assignment under the real-time traffic scenarios which is the key technology in ION [02].

This paper presents an improved ant colony optimization algorithm which can solve the dynamic RWA problem in ION where the nodes have no wavelength conversion capabilities. Section 2 gives introductions of state-of-art RWA solutions. Section 3 presents the principle of the improved algorithm. Simulation and analysis are given in Section 4.

\section{Routing AND WAVELENGTH ASSIGNMENT}

It has been proved that RWA is a NP-C problem [03], and most of the existing RWA solutions can be divided into two common types:

(1) Divide the RWA problem into two independent subproblems (routing and wavelength assignment), then solve the two sub-problems separately.

Generally, routing algorithm consists of three major types: fixed routing (FR) algorithm, fixed-alternative routing (AFR) algorithm and adaptive routing (AR) algorithm [04]. FR algorithm only calculates single route for connection establishment; AFR calculates multiple routes and select an available route with a fixed order; AR also pre-calculates multiple routes, while comparing and selecting the best path depending on some expected optimal goals. Major wavelength assignment algorithms include: Randomly Assigned algorithm (RA), First Fit algorithm (FF), Relative Capacity Loss algorithm (RCL) and Relative Lease Influence (RLI) algorithm, respectively. The similar ideas of these algorithms are how to select the best path (wavelength) under the wavelength continuity constraint. The RA and FF algorithms are popular due to the relative low difficulty. However, it is difficult to get the global optimal solution while solving the routing and wavelength assignment subprolems independently.

(2) Parallel computing using layered graph model (LG). The LG model maps the physical topology into several layered graph models for calculate the wavelength selection and assignment parallel. The advantages of LG model is the 
simplification of dynamic RWA problem, but the shortcomings are the enlargement of the network scale by $|\mathrm{W}|$ times $(|\mathrm{W}|$ equals the number of wavelength on the fiber channel) which need much more computing time [05].

Some evolution algorithms have been posed to solve the dynamic RWA problem in ION including simulated annealing algorithm, swarm intelligence algorithm, genetic algorithm, etc. Here we introduce an improved ant colony based dynamic DBA algorithm.

\section{ALGORITHM DESCRIPTION}

Ant colony optimization algorithm (ACO) was first proposed by M. Dorigo and V. Maniezzo which is inspired based on the behavior of ants seeking a path between their colony and a source of food [06]. The original idea of ACO algorithm has diversified to solve a wider class of numerical problems, e.g. scheduling problem, assignment problem, routing problem, respectively. ACO algorithm is essentially a swarm optimization based heuristic searching algorithm due to its distributed cooperative and positive feedback characteristics. ACO algorithm makes full use of the transfer of biological information between different individuals to find the optimal (shortest) path from the nest to the food source. Based on ACO algorithms, Dorigo and Gamberdella also proposed ant colony system (Ant Colony System, ACS) to improve the algorithm performance [07].

Early ACO algorithms neglect the wavelength assignment and network load balancing while using ACO to solve routing computing. To solve the above shortcomings we propose an improved ACO algorithm whose idea is introducing link idle into the ant transfer probability as a new constraint. At the same time, the random disturbance is added to prevent premature convergence into local optimal path. Each ant is responsible for both routing and wavelength assignment task simultaneously in our improved algorithm, i.e. on each node, each ant should first determine wavelength availability, then calculate expected route, which combines routing and wavelength assignment together. In the addition, each ant is needless to return to the starting point while it arriving the target node which can reduce the total searching time. On the other side, ant should suicide if there is no available routing to present algorithm falling into endless loop.

Some definition are introduced as follows:

(1) $\tau i j w(t)$ is the quantity of pheromone on link $(i, j)$ using wavelength $\mathrm{W}$ at time $\mathrm{t}$.

(2) I ijw(t) indicates the idle probability of wavelength W between link $(i, j)$ at time $t$, which is expressed as

$$
I_{\mathrm{ijw}}(\mathrm{t})=\frac{\left|\mathrm{N}_{\mathrm{ijw}}\right|-\mathrm{n}_{\mathrm{ijw}}(\mathrm{t})}{\left|\mathrm{N}_{\mathrm{ijw}}\right|}
$$

Here $|\mathrm{Nijw}|$ is the total number of wavelength $\mathrm{W}$ between link $(i, j)$, nijw $(t)$ indicates actual wavelength occupation $\mathrm{W}$ between link $(i, j)$ at time $t$.

(3) For ant $\mathrm{k}$, the transfer probability from node $\mathrm{i}$ to node $\mathrm{j}$ at time $t$ can be expressed as

$$
P_{i j}^{k}(t)= \begin{cases}\frac{\left\{\tau_{i j w}^{\alpha}(t) \eta_{i j}^{\beta}(t)+e^{I_{j o w}(t)-1}\right\} \cdot I_{i j w}(t)}{\sum_{s \in a l l o w e d}\left\{\tau_{i s w}^{\alpha}(t) \eta_{i s}^{\beta}(t)+e^{I_{i s w}(t)-1}\right\} \cdot I_{i s w}(t)} & j \in \text { allowed }_{k} \\ 0 & \text { otherwise }\end{cases}
$$

Here, $\alpha \geqslant 0$ is the heuristic factor of pheromone which presents the importance of the trail; $\eta \mathrm{ij}(\mathrm{t})$ is the heuristic function of link (i, j), $\beta \geqslant 0$ is the inspired factor of expectation which indicate the importance of visibility; allowedk represents the available node set at time $t$ (allowedk $=\mathrm{C}$-Tabuk, $\mathrm{C}$ is the node set which have link with node $\mathrm{i}$, Tabuk indicates the traversing node set at time tin this searching cycle) .

(4)Considering the real-time service requirements, a practical dynamic RWA algorithm should not have too much iteration generations. Thus, a random disturbance needs to be introduced in order to prevent algorithm convergence to local optimization prematurely. The random disturbance is implemented by follows: at node $r$,each ant selecting next node s obey the rule defined in equation (3).

$$
s= \begin{cases}\arg \max _{\text {ueallowed }}\left\{\left[\left[\tau_{\text {rnw }}(t)^{\alpha} \cdot\left[\eta_{r u}\right]^{\beta}\right]\right\}+e^{I_{n n w}(t)-1}\right\} \cdot I_{\text {rnw }}(t) & q \leq q_{0} \\ \text { Random select path } & q_{0}<q \leq 1\end{cases}
$$

Here, $\mathrm{q}$ is a random number with uniformly distributed between $[0,1], \mathrm{q} 0$ and $\mathrm{q} 1$ are parameters for random disturbance where $(0 \leq \mathrm{q} 0 \leq 1, \mathrm{q} 0 \leq \mathrm{q} 1 \leq 1)$.

The flow diagram of improved ACO algorithm is shown in Figure 1.

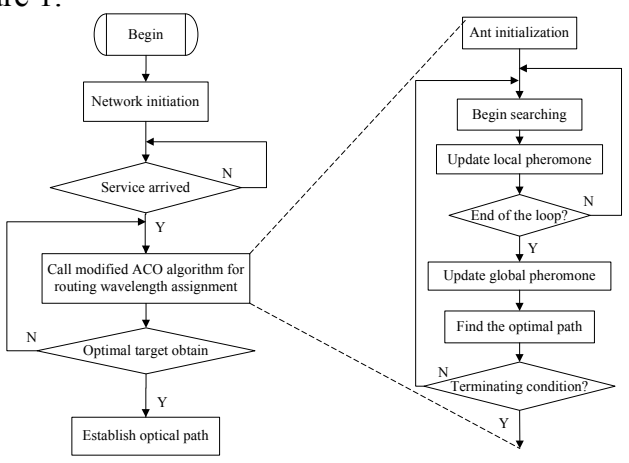

Figure 1. Flow diagram of improved ACO algorithm

\section{Simulation AND ANALYSIS}

Computer simulation is used to compare the performance of improved ACO algorithm with traditional Dijkstra + FF algorithm. Network topology in simulation is the 14 node NSFNET network shown in Figure 2 [08]. There are four link between each nodes which has up to six available wavelength. 


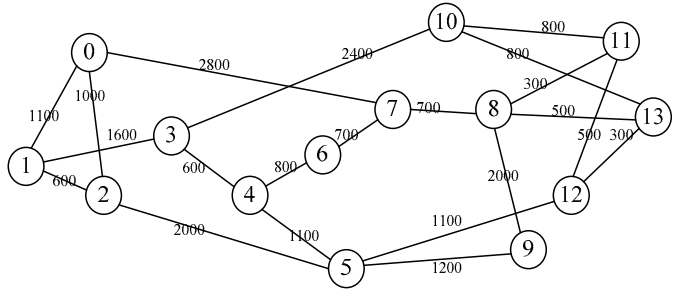

Figure 2. Simulation topology

In our simulation, service requests arrive randomly with Poisson distribution. Once the service request refused, it will be discarded immediately, i.e. none waiting queue existing. All the nodes in our simulation have not configured wavelength conversion function. Simulation parameters set as follows: $\mathrm{Q}=100, \alpha=0.999, \beta=5.0, \rho=0.5, \alpha 1=0.15, \mathrm{q} 0=0.1$, $\mathrm{q} 1=0.25$, number of ant is 10 and the number of iterations is 200. Simulation results are shown in Figure 3 and 4.

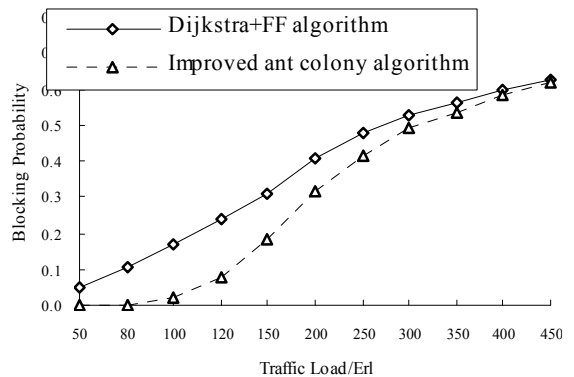

Figure 3. Blocking Probability Comparison

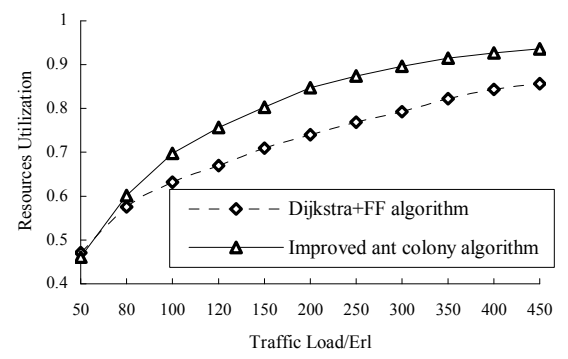

Figure 4. Resource Utilization Comparisons
Simulation results show that improved ACO algorithm has very low blocking probability compared with the Dijkstra + FF algorithm when the traffic load is small. Moreover, improved ACO algorithm has significant better performance both the blocking probability and network resource utilization compared with the Dijkstra + FF algorithm.

\section{CONCLUSIONS}

ACO algorithm is widely accepted as a promising tool to many complex optimization problems due to its good global optimization capability and parallel computing features. This paper presents an improved ACO algorithm by introducing the link idle and random disturbance as constraint to solve the RWA problem in ION. Simulation shows that the modified algorithm decreases the blocking probability and increases the resources utilization comparing with the traditional Dijkstra+FF algorithm, respectively.

\section{REFERENCES}

[1] JAJSZCZYK A. Automatically Switched Optical Networks: Benefits and Requirements [J].IEEE Optical Communication 2005:10-15.

[2] ITU-T Rec.G.8080/Y.1304.Architecture for the Automatically Switched Optical Network (ASON)[S], 2001.

[3] ZHENG Yan-lei, GU Wan-yi, HUANG Shan-guo, ZHANG Pei. An Ant-Based Research on RWA in Optical Networks[C]. 2009 International Conference on Electronic Computer Technology,2009:73-76.

[4] Yi Xie. Research and Simulation on Routing Problems in Intelligent Optical Networks [D]. Beijing University of Posts and Telecommunications. 2006

[5] Shizhong Xu,Lemin Li,and Sheng Wang. Dynamic Routing and Assignment of Wavelength Algorithms in Multifiber Wavelength Division Multiplexing Network[J]. IEEE JOURNAL ON SELECTED AREAS IN COMMUNICATIONS, 2000,18(10):21302137

[6] M. Dorigo, Optimization, Learning and Natural Algorithms. Ph.D.Thesis, Politecnico di Milano, Italy, [in Italian], 1992

[7] Marco Dorigo,Luca Maria Gambardella. Ant Colony System: A Cooperative Learning Approach to the raveling Salesman Problem [J]. IEEE Transactions on Evolutionary Computation,1997,1 (1) : 532556.

[8] Joan Triay,Cristina Cervelló-Pastor.An Ant-Based Algorithm for Distributed Routing and Wavelength Assignment in Dynamic Optical Network[J]. IEEE JOURNAL ON SELECTED AREAS IN COMMUNICATIONS, VOL. 28, NO. 4, MAY 2010:542-552. 